\title{
Modulatory effect of silymarin on pulmonary vascular dysfunction through HIF-1 $\alpha$-iNOS following rat lung ischemia-reperfusion injury
}

\author{
YANWU JIN ${ }^{1}$, XIN ZHAO $^{1}$, HE ZHANG $^{1}$, QINGSONG LI ${ }^{1}$, GUODONG LU $^{1}$ and XIAOGANG ZHAO ${ }^{2}$ \\ Departments of ${ }^{1}$ Anesthesiology II and ${ }^{2}$ Thoracic Surgery, Second Hospital of Shandong University, \\ Jinan, Shandong 250033, P.R. China
}

Received April 10, 2015; Accepted November 25, 2015

DOI: $10.3892 / \mathrm{etm} .2016 .3370$

\begin{abstract}
Silymarin is a traditional therapeutic used to protect the liver, acting to oppose lipid peroxidation, to enhance liver regeneration and functioning as an antioxidant. However, the effects of silymarin on pulmonary vascular dysfunction have not been investigated. In the present study, the modulatory effects of silymarin on pulmonary vascular dysfunction and the underlying mechanisms behind this were investigated in a lung ischemia-reperfusion (I/R) injury rat model. Male Sprague Dawley rats were randomly divided into 3 groups, including: i) A control group $(n=10)$; ii) an I/R group $(n=10)$; and iii) a silymarin-treated group $(n=10)$. All experimental rats received $250 \mathrm{mg} / \mathrm{kg} /$ day of silymarin for 8 days. Silymarin was demonstrated to markedly improve lung I/R-induced pulmonary vascular dysfunction and lung moisture. Following silymarin treatment, inflammation and oxidative stress in the lung I/R-injury rats were demonstrably suppressed. Treatment with silymarin also inhibited the activation of caspase-3 and -9 , and hypoxia inducible factor- $1 \alpha(\mathrm{HIF}-1 \alpha)$ and inducible nitric oxide synthase (iNOS) protein expression in the lung I/R-injury rats. Silymarin was concluded to impact upon pulmonary vascular dysfunction through the HIF-1 $\alpha$-iNOS pathway in the lung I/R injury rat model.
\end{abstract}

\section{Introduction}

Lung ischemic-reperfusion (I/R) injury typically occurs as a result of lung transplantation, pulmonary thromboembolism

Correspondence to: Professor Xin Zhao, Department of Anesthesiology II, Second Hospital of Shandong University, 247 Beiyuan Street, Jinan, Shandong 250033, P.R. China

E-mail: lujnzx@sohu.com

Professor Xiaogang Zhao, Department of Thoracic Surgery, Second Hospital of Shandong University, 247 Beiyuan Street, Jinan, Shandong 250033, P.R. China

E-mail: xiaogangzhaozhao@163.com

Key words: silymarin, pulmonary vascular dysfunction, lung ischemia-reperfusion injury, HIF-1 $\alpha$-iNOS resection, pulmonary embolism following thrombolysis treatment and numerous other types of extracorporeal circulation cardiac surgery; I/R is an early post-operative complication, and an important cause of mortality (1). Lung transplantation is the last-resort treatment option for patients with end-stage lung disease, but following lung transplantation, the patient 1-year survival rate is only $\sim 70 \%$; patients tend to succumb to post-operative mortality within 30 days of surgery (2). Graft failure following transplantation can cause lung I/R injury, including injury to the pulmonary vascular endothelium, resulting in marked lung damage (3).

The complex etiology of pulmonary vascular dysfunction has not been fully elucidated; previous studies have associated this disease with oxidative damage caused by oxygen free radicals, calcium overload and neutrophil recruitment caused by excessive inflammation. These physiological effects may produce a greater number of oxygen free radicals than are removed, causing an exacerbation of lung I/R injury (4). A previous study associated hypoxia inducible factor- $1 \alpha(\mathrm{HIF}-1 \alpha)$ and inducible nitric oxide synthase (iNOS) with pulmonary hypertension; furthermore, structural damage to the pulmonary vascular endothelium was also considered to be an initial contributor to pulmonary vascular dysfunction (5).

Silymarin is an active element extracted from Silybum marianum, by 4 isomers of the original ketone group dihydrogen color, including Silybum marianum, has the main pharmacological effects (6). Silymarin can eliminate reactive oxygen species, protect against lipid peroxidation, maintain cell membrane fluidity, protect liver cell membranes and promote liver cell repair and regeneration, which all contribute to combat liver fibrosis (7). The present study aimed to investigate the effects of silymarin on pulmonary vascular dysfunction in a lung I/R injury rat model and assess its underlying molecular mechanism of action.

\section{Materials and methods}

Animals. All experimental procedures conformed to the Guide for the Care and Use of Laboratory Animals (Shandong University, Shandong, China) and were approved by the Institutional Ethics Committee of Shandong University. Male 
Sprague Dawley (SD) rats (180-220 g) were obtained from the Laboratory Animal Center of Shandong University, and were housed in units maintained at appropriate environmental conditions, with access to food and water ad libitum.

Lung I/R injury. SD rats were administered $0.1 \mathrm{mg} / \mathrm{kg} / \mathrm{min}$ of pentobarbital by intraperitoneal injection. Following endotracheal intubation, mechanical ventilation with $100 \%$ oxygen was applied, with a tidal volume of $10 \mathrm{ml} / \mathrm{kg}$ (70 breaths/min). A midline thoracotomy was performed under sterile conditions, the pericardium was opened, and then the left pulmonary artery was ligated using an 8-0 prolene suture and was secured with polyethylene tubing without damaging the vessel. After $1 \mathrm{~h}$ of ischemia, the left pulmonary artery occlusion was cleared and the artery was reperfused with blood. The chest cavity was then closed, and SD rats were restored to normal respiration. Following $24 \mathrm{~h}$ of reperfusion, the rats were sacrificed by decollation following anesthetization.

Experimental design. SD rats were randomly divided into 3 groups, as follows: i) Control group rats $(n=10)$, administered saline solution; ii) I/R group rats $(n=10)$, receiving the aforementioned lung I/R injury and administered saline solution; iii) silymarin group rats $(n=10)$, receiving lung $I / R$ injury and administered $250 \mathrm{mg} / \mathrm{kg} /$ day of silymarin (Sigma-Aldrich, St. Louis, MO, USA) for 8 days (8).

Vascular reactivity. Vascular reactivity was recorded with a myograph, as previously described (9). Briefly, the pulmonary arteries were dissected from the rats and the connective tissue was removed. The pulmonary arteries and surrounding endothelium were maintained in Krebs solution and a myograph was used to estimate the tension in relation to internal circumference. The functional integrity of the pulmonary artery tissue segments was confirmed using $60 \mathrm{mmol} / 1 \mathrm{KCl}$ after $60 \mathrm{~min}$ of stabilization. The endothelium function was evaluated by measuring the relaxant response of segments that had been contracted with $1 \mu \mathrm{mol} / 1$ phenylephrine (Wuhan Xinxin beauty Biotechnology, Co., Ltd., Wuhan, China) to $1 \mu \mathrm{mol} / 1$ acetylcholine (Wuhan Dong Kangyuan Technology, Co., Ltd., Wuhan, China).

Mass calculations. Lung tissue samples were weighed to calculate wet mass; they were then placed in a drying oven at $80^{\circ} \mathrm{C}$ for $12-36 \mathrm{~h}$ and the corresponding dry weight was also recorded. Lung moisture was calculated as the wet/dry weight ratio.

Western blotting. Following treatment with silymarin, lung tissue samples were homogenized with radioimmunoprecipitation assay lysis buffer (Beyotime Institute of Biotechnology, Jiangsu, China). The homogenized samples were centrifuged at $1,200 \mathrm{x} \mathrm{g}$ for $10 \mathrm{~min}$ at $4^{\circ} \mathrm{C}$. The total protein in each supernatant was quantified using a bicinchoninic acid assay (Beyotime Institute of Biotechnology). Equal protein was separated by $10 \%$ sodium dodecyl sulfate-polyacrylamide gel electrophoresis and transferred to nitrocellulose membranes at $100 \mathrm{~V}$ for 1-2 $\mathrm{h}$. The membranes were blocked with phosphate-buffered saline containing $5 \%$ skimmed milk powder for $1-2 \mathrm{~h}$ at room temperature to prevent non-specific binding. The membranes were incubated with primary antibodies against HIF-1 $\alpha$ (1:1,000; C-19; Santa Cruz Biotechnology, Inc., Dallas, TX, USA), iNOS (1:1,000; sc-49058; Santa Cruz Biotechnology, Inc.) and $\beta$-actin (1:500; D110007; Sangon Biotech Co. Ltd., Shanghai, China) overnight at $4^{\circ} \mathrm{C}$. The membranes were incubated with secondary antibodies (1:5,000, BestBio Inc, Shanghai, China) for 2-3 h, then developed using chemiluminescence detection (BestBio Inc.). Relative band density was measured using Multi-Analyst software (Bio-Rad Laboratories, Inc., Hercules, CA, USA).

Inflammatory cytokine levels. The supernatant from centrifuged, homogenized lung tissue samples was acquired and the serum levels of nuclear factor- $\kappa \mathrm{B}(\mathrm{NF}-\kappa \mathrm{B})$, tumor necrosis factor- $\alpha$ (TNF- $\alpha$ ), interleukin (IL)-1 $\beta$ and IL-6 were analyzed using ELISA kits (KF008, R019 and H002, respectively; Nanjing Jiancheng Bioengineering Institute, Nanjing, China), according to the manufacturer's protocols.

Oxidative stress. The supernatant from centrifuged, homogenized lung tissue samples was acquired and the serum superoxide dismutase (SOD) and malondialdehyde (MDA) levels were analyzed using ELISA kits (A001-3 and A003-1, respectively; Nanjing Jiancheng Bioengineering Institute), according to the manufacturer's protocols.

Caspase-9 and caspase-3 levels. The supernatant from centrifuged, homogenized lung tissue samples was acquired and the total protein was quantified using the bicinchoninic acid assay. Equal protein $(30 \mu \mathrm{g})$ across samples was incubated with reaction buffer (Ac-DEVD-pNA to detect caspase-3 or Ac-LEHD-pNA to detect caspase-9) at $37^{\circ} \mathrm{C}$ for $2 \mathrm{~h}$ in the dark, and caspase-3 and -9 levels were measured at an absorbance of $405 \mathrm{~nm}$.

Statistical analysis. Data are presented as the mean \pm standard error of the mean. Statistical analyses, including a one-way analysis of variance with Student's t-test post-hoc analysis, were conducted using SPSS 15.0 statistical software (SPSS, Inc., Chicago, IL, USA). $\mathrm{P}<0.05$ was considered to indicate a statistically significant difference.

\section{Results}

Silymarin improves I/R-induced pulmonary vascular dysfunction. The chemical structure of silymarin is indicated in Fig. 1 (Sigma-Aldrich Chemie Gmbh, Munich, Germany). To determine whether silymarin affects lung I/R-induced pulmonary vascular dysfunction in rats, vascular reactivity was evaluated following reperfusion. Compared with the control group, the relaxant response was effectively decreased in lung I/R-injured rats ( $\mathrm{P}<0.01$; Fig. 2). However, treatment with silymarin improved the relaxant response in lung I/R-injured rats, when compared with that of the saline-treated lung I/R-injured group ( $\mathrm{P}<0.01$; Fig. 2).

Silymarin reduces $I / R$-induced lung moisture. The effects of silymarin on lung moisture following I/R injury were also evaluated. As indicated in Fig. 3, lung moisture was significantly higher in the I/R-injured group than the control 
<smiles>COc1cc([C@H]2Oc3c(O)cc([C@@H]4Oc5cc(O)cc(O)c5C(=O)[C@H]4O)cc3[C@H]2CO)ccc1O</smiles>

Figure 1. Chemical structure of silymarin.

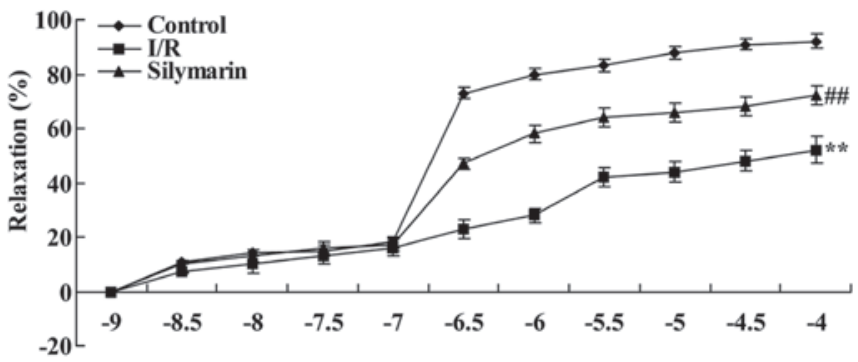

Figure 2. Silymarin increases I/R-induced pulmonary vascular dysfunction. ${ }^{* * *} \mathrm{P}<0.01$ compared with the control group; ${ }^{\# \#} \mathrm{P}<0.01$ compared with the I/R group. I/R, ischemia/reperfusion.

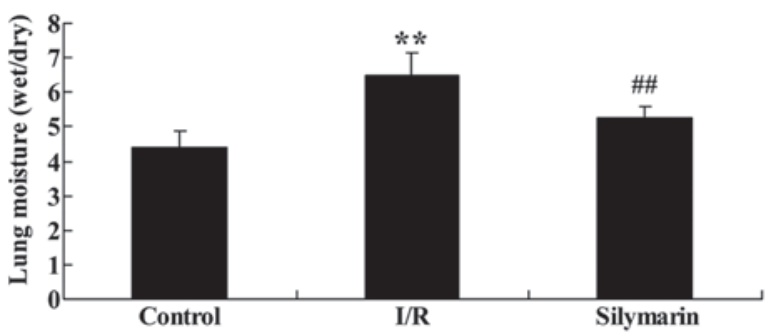

Figure 3. Silymarin reduces I/R-induced lung moisture. ${ }^{* *} \mathrm{P}<0.01$ compared with the control group; ${ }^{\#} \mathrm{P}<0.01$ compared with the $\mathrm{I} / \mathrm{R}$ group. $\mathrm{I} / \mathrm{R}$, ischemia/reperfusion.

group $(\mathrm{P}<0.01)$, but treatment with silymarin markedly reduced lung moisture compared with the I/R-injured group $(\mathrm{P}<0.01)$.

Silymarin reduces I/R-induced inflammation of the lungs. In order to investigate the inflammatory effects of silymarin, the serum NF- $\kappa \mathrm{B}, \mathrm{TNF}-\alpha$, IL-1 $\beta$ and IL- 6 protein levels were analyzed following reperfusion. These inflammatory factors were significantly increased in I/R-injured rats compared with the control group $(\mathrm{P}<0.01$; Fig. 4). However, protein levels of these inflammatory factors were significantly reduced following silymarin treatment of I/R-injured rats $(\mathrm{P}<0.01$; Fig. 4).

Silymarin increases SOD and reduces MDA levels in rat lungs following I/R injury. The anti-oxidative effects of silymarin were also examined, using the serum SOD and MDA levels. Serum SOD levels were significantly decreased and serum MDA levels were significantly increased in the lungs of rats following I/R injury, as compared with the control group $(\mathrm{P}<0.01$; Fig. 5). However, serum levels of SOD were
A

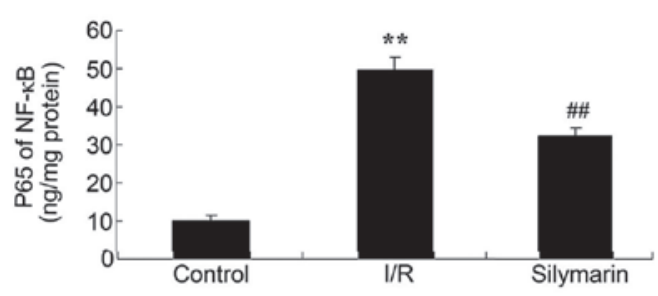

B

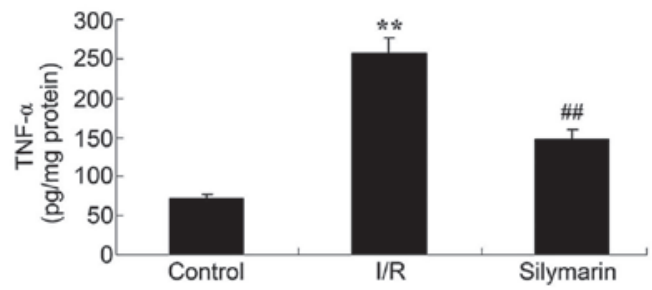

C

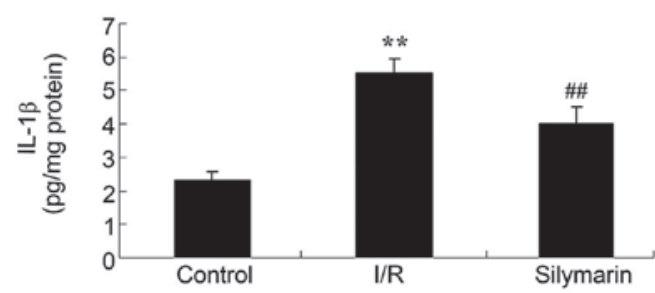

D

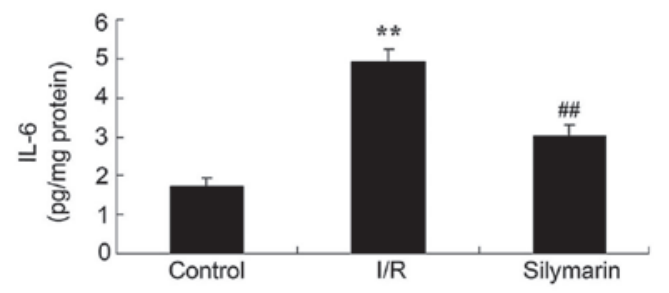

Figure 4. Silymarin reduces I/R-induced inflammation in the lungs, as evaluated by serum levels of (A) NF- $\kappa$ B, (B) TNF- $\alpha$, (C) IL- $1 \beta$ and (D) IL-6. ${ }^{* *} \mathrm{P}<0.01$ compared with the control group; ${ }^{\# \#} \mathrm{P}<0.01$ compared with the $\mathrm{I} / \mathrm{R}$ group. I/R, ischemia/reperfusion; NF- $\kappa B$, nuclear factor $\kappa B$; TNF- $\alpha$, tumor necrosis factor- $\alpha$; IL, interleukin.

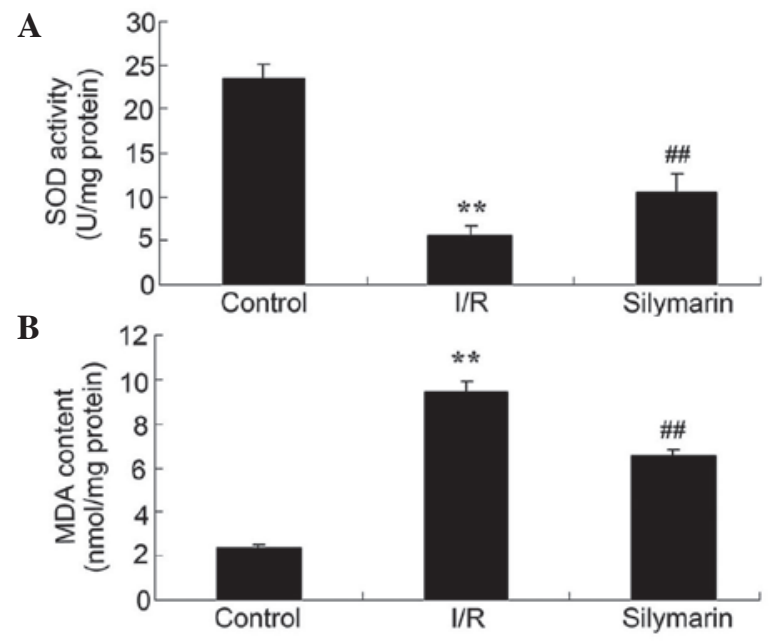

Figure 5. Silymarin increases (A) SOD and reduces (B) MDA levels in the lungs of rats following $\mathrm{I} / \mathrm{R}$ injury. ${ }^{* *} \mathrm{P}<0.01$ compared with the control group; ${ }^{\# \#} \mathrm{P}<0.01$ compared with the $\mathrm{I} / \mathrm{R}$ group. I/R, ischemic/reperfusion; SOD, superoxide dismutase; MDA, malondialdehyde.

significantly increased and serum levels of MDA were significantly decreased in the lungs of silymarin-treated rats, as compared with the I/R-injured group ( $\mathrm{P}<0.01$; Fig. 5). 

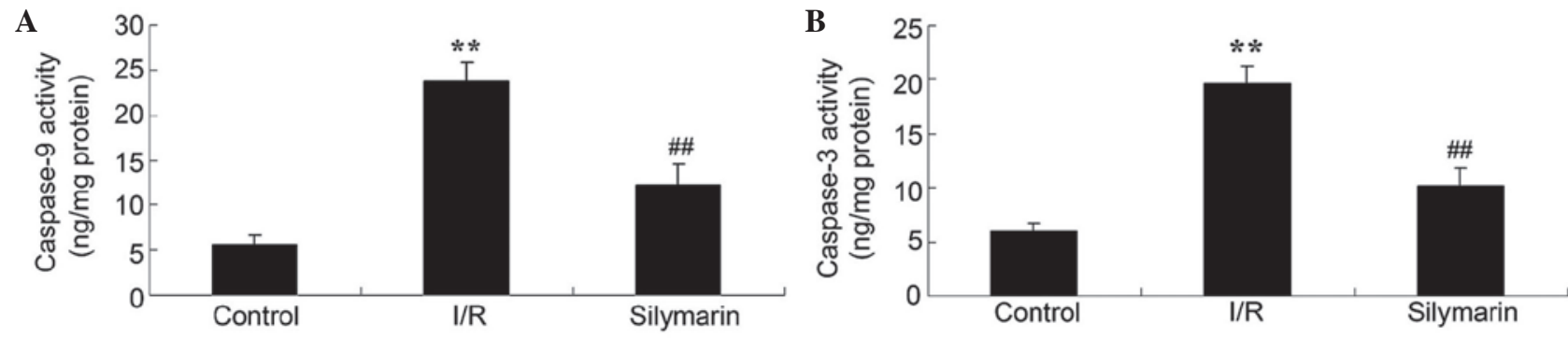

Figure 6. Silymarin prevents I/R-induced cell apoptosis in the lungs, as measured by (A) caspase- 9 and (B) caspase-3 levels. ${ }^{* * *} \mathrm{P}<0.01$ compared with the control group; ${ }^{\# \#} \mathrm{P}<0.01$ compared with the I/R group. I/R, ischemia/reperfusion.

A

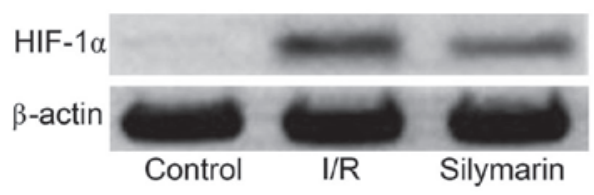

B

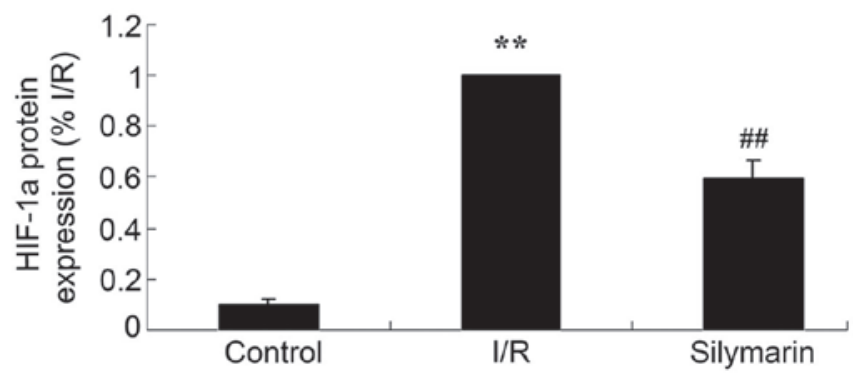

Figure 7. Silymarin reduces I/R-induced HIF-1 $\alpha$ expression in the lungs, evaluated using (A) western blotting assays and (B) statistical analysis of HIF-1 $\alpha$ expression levels. ${ }^{* *} \mathrm{P}<0.01$ compared with the control group; ${ }^{\# \#} \mathrm{P}<0.01$ compared with the I/R group. HIF-1 $\alpha$, hypoxia inducible factor-1 $\alpha$; $/ \mathrm{R}$, ischemia/reperfusion.

A

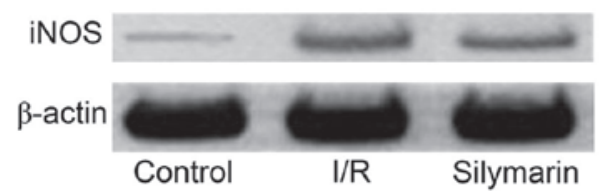

B

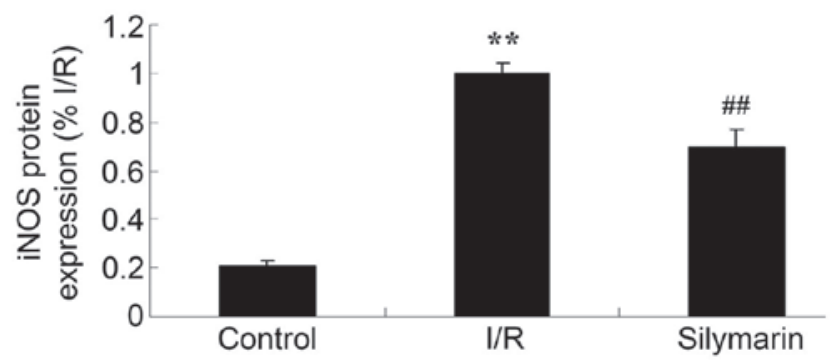

Figure 8. Silymarin attenuates I/R-induced iNOS expression in the lungs, as evaluated using (A) western blotting assays and (B) statistical analysis of iNOS expression levels. ${ }^{* *} \mathrm{P}<0.01$ compared with the control group; ${ }^{\# \#} \mathrm{P}<0.01$ compared with the I/R group. iNOS, inducible nitric oxide synthase; I/R, ischemia/reperfusion.

Silymarin ameliorates $I / R$-induced cell apoptosis in the lungs. In order to elucidate the effect of silymarin on cell apoptosis following lung I/R, the levels of caspase-3 and caspase-9 were investigated following reperfusion. Lung I/R injury markedly increased the caspase-3 and -9 levels compared with the control group $(\mathrm{P}<0.01$; Fig. 6). However, treatment with silymarin markedly decreased caspase-3 and -9 levels compared with the I/R-injured group $(\mathrm{P}<0.01$; Fig. 6).

Silymarin attenuates $I / R$-induced HIF-1 $\alpha$ expression in the lungs. In order to reveal the effects of silymarin on HIF-1 $\alpha$ expression, HIF-1 $\alpha$ protein expression was analyzed using western blotting. HIF-1 $\alpha$ protein levels were increased subsequent to I/R injury compared with the control group $(\mathrm{P}<0.01$; Fig. 7). As demonstrated in Fig. 7, HIF-1 $\alpha$ protein levels were decreased following silymarin treatment of lung I/R-injured rats $(\mathrm{P}<0.01$; Fig. 7$)$.
Silymarin reduces $I / R$-induced iNOS expression in the lungs. The effects of silymarin treatment on iNOS expression were examined using western blotting; this revealed that the protein expression level of iNOS was increased following I/R injury compared with the control group $(\mathrm{P}<0.01$; Fig. 8). However, silymarin treatment decreased iNOS expression compared with that in lung I/R-injured rats $(\mathrm{P}<0.01$; Fig. 8).

\section{Discussion}

Lung I/R injury refers to restore blood flow perfusion, on the basis of ischemia of lung tissue damage is aggravating pathological state. In numerous situations known to cause lung I/R injury, including thrombolysis for pulmonary embolism, interventional therapy and lung transplantation, the clinical focus is the prevention of lung I/R injury (10). The major pathological changes following I/R injury are as follows: i) Intimal hyperplasia of the pulmonary artery, increased elastic fibers 
and collagen matrix, hardening of blood vessels and increased resistance of the blood vessels; ii) hyperplasia and hypertrophy of the smooth muscle cell membrane, leading to intermembrane hypertrophy; and iii) loss of the muscular layer of the pulmonary arteriole (11). The present study demonstrated that silymarin significantly reduced lung I/R-induced pulmonary vascular dysfunction and lung moisture. Concordantly, Demir et al (12) revealed that silymarin attenuates mesenteric I/R injury, and Turgut et al (13) reported that silymarin protects against kidney I/R injury. The use of silymarin may therefore be considered a novel therapeutic strategy for protection against lung I/R injury.

Animal and clinical trials have confirmed that the inflammatory response is focal to continuing tissue damage following cerebral ischemia, and this is observed to a greater degree following lung I/R injury $(14,15)$. Reduction in the inflammatory response is therefore an important strategy to attenuate lung I/R injury. NF- $\kappa \mathrm{B}$ is an important signal transduction molecule in the inflammatory response, with a central role in the inflammatory response to lung I/R injury $(16,17)$. Activation of the $N F-\kappa B$ pathway induces the expression of adhesion molecules and chemokines, and the expression of pro-inflammatory factors; this response leads to leukocyte adhesion, aggregation and infiltration in the ischemic region, aggravating lung I/R injury (18). In the present study, silymarin significantly reduced the serum NF- $\kappa \mathrm{B}, \mathrm{TNF}-\alpha, \mathrm{IL}-1 \beta$ and IL-6 levels in the lungs of I/R-injured rats. A previous study demonstrated that silymarin suppressed inflammation in mycobacterial adjuvant-induced arthritis (19) and in the skin of SENCAR mice (20). Silymarin may therefore represent a potent agent for protection against the lung I/R-induced inflammatory response.

Lung I/R injury is a significant cause of post-operative acute lung injury and multiple organ failure. Oxidative stress is central to I/R injury; a previous study reported that the majority of lung tissue cells generate damaging reactive oxygen species, and that the abundant capillaries and fragile wall structure of the lungs makes them vulnerable to oxidative stress (21). MDA is a terminal product of lipid peroxidation, such that its expression levels reflect the degree of lipid peroxidation damage (22). Furthermore, SOD and catalase are important antioxidant enzymes of the cell; the levels of which reflect the protective ability of lung tissue cells (23). Therefore, oxidative stress is may be considered important in the process of lung I/R injury (22). The data from the present study demonstrated that silymarin significantly increased the serum levels of SOD in rats with lung I/R injuries. A previous study by Clichici et al (24) reported that silymarin inhibited the progression of fibrosis through suppression of oxidative stress, and Moshtaghion et al (25) revealed that silymarin prevented varicocele-induced damage through an increase in MDA expression. The beneficial effects of silymarin may therefore be attributed to anti-oxidative effects.

HIF- $1 \alpha$ is a transcription factor involved in altering cellular oxygen concentration and regulation of gene expression (26). The lung I/R injury-induced HIF-1 $\alpha$ expression observed in the present study likely exacerbated the injury of lung tissue cells, which is consistent with the results of a previous study (27). HIF-1 $\alpha$ promotion of lung tissue cell injury may be associated with hypoxia; the mitochondria of I/R-conditioned cells produce large amounts of reactive oxygen species, in addition to the hydroxide produced during I/R (28). A previous study in a rat model of pulmonary hypertension reported increased HIF-1 $\alpha$ expression in the pulmonary artery intima, raising the level of downstream iNOS expression; this resulted in the proliferation of vascular endothelial cells within the pulmonary artery intima and concomitant structural damage to the pulmonary vascular endothelium, thereby promoting angiogenesis and pulmonary vascular remodeling (29). This prior study indicated that, at the protein and gene level, HIF- $1 \alpha$ and iNOS may contribute to hypoxia, causing lung I/R injury (4). In the present study, silymarin significantly reduced caspase-3 and -9, HIF-1 $\alpha$ and iNOS expression levels in rats following lung I/R injury. Similarly, Li et al (30) previously reported that silymarin reduced the UV-irradiated caspase-3 and -9 activities in A375-S2 cells, and Atawia et al (31) demonstrated that silymarin reduced the production of inflammatory mediators by downregulation of HIF-1 $\alpha$, iNOS and NF- $\kappa \mathrm{B}$. Kim et al (32) demonstrated that silymarin inhibits NO and iNOS production in pancreatic $\beta$ cells. Therefore, the regulatory effect of silymarin against lung I/R injury may be associated with the caspase/HIF-1 $\alpha /$ iNOS pathway.

In conclusion, the present study demonstrated that silymarin treatment was able to improve pulmonary vascular dysfunction following lung I/R injury via the HIF-1 $\alpha$-iNOS pathway. Further studies are required in order to elucidate the protective effects of silymarin against lung I/R injury.

\section{Acknowledgements}

The present study was supported by the Natural Science Foundation of Shandong Province (grant no.ZR2012HL54 and ZR2013HL022) and the Seed Fund of The Second Hospital of Shandong University (grant no. S2015010018).

\section{References}

1. Mansour Z, Charles AL, Kindo M, Pottecher J, Chamaraux-Tran TN, Lejay A, Zoll J, Mazzucotelli JP and Geny B: Remote effects of lower limb ischemia-reperfusion: Impaired lung, unchanged liver, and stimulated kidney oxidative capacities. BioMed Res Int 2014: 392390, 2014.

2. Diao TJ, Chen X, Deng LH, Chen HX, Liang Y, Zhao XD, Wang QH, Yuan WS, Gao BC and Ye Y: Protective effect of nitric oxide on hepatopulmonary syndrome from ischemia-reperfusion injury. World J Gastroenterol 18: 3310-3316, 2012.

3. Lv X, Tan J, Liu D, Wu P and Cui X: Intratracheal administration of $\mathrm{p} 38 \alpha$ short-hairpin RNA plasmid ameliorates lung ischemia-reperfusion injury in rats. J Heart Lung Transplant 31: 655-662, 2012

4. Zhao X, Jin Y, Li H, Wang Z, Zhang W and Feng C: Hypoxia-inducible factor 1alpha contributes to pulmonary vascular dysfunction in lung ischemia-reperfusion injury. Int J Clin Exp Pathol 7: 3081-3088, 2014.

5. Natarajan R, Jones DG, Fisher BJ, Wallace TJ, Ghosh S and Fowler AA III: Hypoxia inducible factor-1: Regulation by nitric oxide in posthypoxic microvascular endothelium. Biochem Cell Biol 83: 597-607, 2005.

6. Malekinejad H, Sheikhzadeh S and Hobbenaghi R: Silymarin attenuates mycophenolate mofetil-induced duodenal disorders in rats. Avicenna J Phytomed 4: 170-181, 2014.

7. Bonifaz V, Shan Y, Lambrecht RW, Donohue SE, Moschenross D and Bonkovsky HL: Effects of silymarin on hepatitis $\mathrm{C}$ virus and haem oxygenase-1 gene expression in human hepatoma cells. Liver Int 29: 366-373, 2009.

8. Rao PR and Viswanath RK: Cardioprotective activity of silymarin in ischemia-reperfusion-induced myocardial infarction in albino rats. Exp Clin Cardiol 12: 179-187, 2007. 
9. Moral-Sanz J, Menendez C, Moreno L, Moreno E, Cogolludo A and Perez-Vizcaino F: Pulmonary arterial dysfunction in insulin resistant obese Zucker rats. Respir Res 12: 51, 2011

10. Luo C, Yuan D, Zhao W, Chen H, Luo G, Su G and Hei Z: Sevoflurane ameliorates intestinal ischemia-reperfusion-induced lung injury by inhibiting the synergistic action between mast cell activation and oxidative stress. Mol Med Rep 12: 1082-1090, 2015.

11. Shoji T, Omasa M, Nakamura T, Yoshimura T, Yoshida H, Ikeyama K, Fukuse T and Wada H: Mild hypothermia ameliorates lung ischemia reperfusion injury in an ex vivo rat lung model. Eur Surg Res 37: 348-353, 2005.

12. Demir M, Amanvermez R, Kamalı Polat A, Karabıçak I, Cinar H, Kesicioğlu T and Polat C: The effect of silymarin on mesenteric ischemia-reperfusion injury. Med Princ Pract 23: 140-144, 2014

13. Turgut F, Bayrak O, Catal F, Bayrak R, Atmaca AF, Koc A, Akbas A, Akcay A and Unal D: Antioxidant and protective effects of silymarin on ischemia and reperfusion injury in the kidney tissues of rats. Int Urol Nephrol 40: 453-460, 2008.

14. Yousefi H, Ahmadiasl N, Alihemmati A and Habibi P: Effect of renal ischemia-reperfusion on lung injury and inflammatory responses in male rat. Iran J Basic Med Sci 17: 802-807, 2014.

15. Chen LF, Tian YF, Lin CH, Huang LY, Niu KC and Lin MT: Repetitive hyperbaric oxygen therapy provides better effects on brain inflammation and oxidative damage in rats with focal cerebral ischemia. J Formos Med Assoc 113: 620-628, 2014.

16. Zhang F, Lu M, Wang H and Ren T: Aspirin attenuates angiotensin II-induced inflammation in bone marrow mesenchymal stem cells via the inhibition of ERK1/2 and NF- $\mathrm{BB}$ activation. Biomed Rep 1: 930-934, 2013.

17. Jin LY, Li CF, Zhu GF, Wu CT, Wang J and Yan SF: Effect of siRNA against NF- $\kappa \mathrm{B}$ on sepsis-induced acute lung injury in a mouse model. Mol Med Rep 10: 631-637, 2014.

18. Amoruso A, Bardelli C, Cattaneo CI, Fresu LG, Manzetti E and Brunelleschi S: Neurokinin (NK)-1 receptor expression in monocytes from bipolar disorder patients: A pilot study. J Affect Disord 178: 188-192, 2015.

19. Gupta OP, Sing S, Bani S, Sharma N, Malhotra S, Gupta BD, Banerjee SK and Handa SS: Anti-inflammatory and anti-arthritic activities of silymarin acting through inhibition of 5-lipoxygenase. Phytomedicine 7: 21-24, 2000.

20. Zhao J, Lahiri-Chatterjee M, Sharma Y and Agarwal R: Inhibitory effect of a flavonoid antioxidant silymarin on benzoyl peroxide-induced tumor promotion, oxidative stress and inflammatory responses in SENCAR mouse skin. Carcinogenesis 21: $811-816,2000$
21. Yao R, Zhou Y, He Y, Jiang Y, Liu P, Ye L, Zheng Z, Lau WB, Cao $Y$ and Zeng Z: Adiponectin protects against paraquat-induced lung injury by attenuating oxidative/nitrative stress. Exp Ther Med 9: 131-136, 2015.

22. Sulkowska M and Sulkowski S: The effect of pentoxifylline on ultrastructural picture of type II alveolar epithelial cells and generation of reactive oxygen species during cyclophosphamide-induced lung injury. J Submicrosc Cytol Pathol 29: 487-496, 1997.

23. Fu Z, Liu X, Geng B, Fang L and Tang C: Hydrogen sulfide protects rat lung from ischemia-reperfusion injury. Life Sci 82: 1196-1202, 2008.

24. Clichici S, Olteanu D, Nagy AL, Oros A, Filip A and Mircea PA: Silymarin inhibits the progression of fibrosis in the early stages of liver injury in $\mathrm{CCl}_{4}$-treated rats. J Med Food 18: 290-298, 2015.

25. Moshtaghion SM, Malekinejad H, Razi M and Shafie-Irannejad V: Silymarin protects from varicocele-induced damages in testis and improves sperm quality: Evidence for E2f1 involvement. Syst Biol Reprod Med 59: 270-280, 2013.

26. Prangsaengtong O, Park JY, Inujima A, Igarashi Y, Shibahara N and Koizumi K: Enhancement of lymphangiogenesis in vitro via the regulations of HIF-1 $\alpha$ expression and nuclear translocation by deoxyshikonin. Evid Based Complement Alternat Med 2013: 148297,2013

27. Kannan KB, Colorado I, Reino D, Palange D, Lu Q, Qin X, Abungu B, Watkins A, Caputo FJ, Xu DZ, et al: Hypoxia-inducible factor plays a gut-injurious role in intestinal ischemia reperfusion injury. Am J Physiol Gastrointest Liver Physiol 300: G853-G861, 2011.

28. Haddad JJ: Science review: Redox and oxygen-sensitive transcription factors in the regulation of oxidant-mediated lung injury: Role for hypoxia-inducible factor-1 alpha. Crit Care 7: 47-54, 2003.

29. Jiang H, Huang Y, Xu H, Hu R and Li QF: Inhibition of hypoxia inducible factor-1 $\alpha$ ameliorates lung injury induced by trauma and hemorrhagic shock in rats. Acta Pharmacol Sin 33: 635-643, 2012

30. Li LH, Wu LJ, Zhou B, Wu Z, Tashiro S, Onodera S, Uchiumi F and Ikejima T: Silymarin prevents UV irradiation-induced A375-S2 cell apoptosis. Biol Pharm Bull 27: 1031-1036, 2004.

31. Atawia RT, Mosli HH, Tadros MG, Khalifa AE, Mosli HA and Abdel-Naim AB: Modulatory effect of silymarin on inflammatory mediators in experimentally induced benign prostatic hyperplasia: Emphasis on PTEN, HIF-1 $\alpha$, and NF- $\mathrm{KB}$. Naunyn Schmiedebergs Arch Pharmacol 387: 1131-1140, 2014.

32. Kim EJ, Kim J, Lee MY, Sudhanva MS, Devakumar S and Jeon YJ: Silymarin inhibits cytokine-stimulated pancreatic beta cells by blocking the ERK1/2 pathway. Biomol Ther (Seoul) 22: 282-287, 2014. 